Research Article

\title{
Modulation and Signal Detection for Diffusive-Drift Molecular Communication with a Mobile Receiver
}

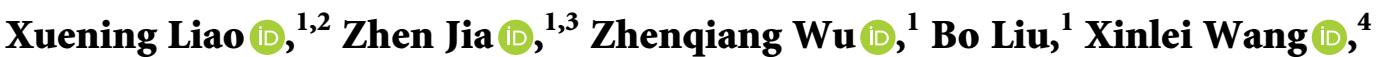 \\ and Xiaohong Jiang ${ }^{5}$ \\ ${ }^{1}$ School of Computer Science, Shaanxi Normal University, Xi'an, Shaanxi 710119, China \\ ${ }^{2}$ Shaanxi Key Laboratory for Network Computing and Security Technology, Xi'an, Shaanxi 710048, China \\ ${ }^{3}$ Collaborative Innovation Center of Assessment Toward China Basic Education Quality, Shaanxi Normal University Branch, \\ Xi'an 710119, China \\ ${ }^{4}$ School of Computer Science, Xi'an Aeronautical University, Xi'an 710077, China \\ ${ }^{5}$ School of Systems Information Science, Future University Hakodate, Kamedanakano-Cho, Hakodate, \\ Hokkaido 041-8655, Japan
}

Correspondence should be addressed to Zhenqiang Wu; zqiangwu@snnu.edu.cn

Received 24 May 2021; Accepted 17 November 2021; Published 2 December 2021

Academic Editor: Alessandro Bazzi

Copyright (C) 2021 Xuening Liao et al. This is an open access article distributed under the Creative Commons Attribution License, which permits unrestricted use, distribution, and reproduction in any medium, provided the original work is properly cited.

Molecular communication (MC), which allows nanomachines to communicate with each other by using chemical molecules, is considered to be a promising method for communications in liquid environment. Available works on MC mainly focus on modulation and signal detection schemes for MC systems with fixed nanomachines, i.e., fixed molecular communication (FMC) systems. However, the more complex systems with mobile nanomachines (i.e., mobile molecular communication (MMC) systems) have been largely unexplored. This paper considers a MMC system with a fixed transmitter and a mobile receiver communicating over diffusive-drift channels of a limited boundary. We first propose a new modulation scheme to address the issue of misalignment in the signal detection of MMC systems by adopting three types of molecules in the signal modulation and modulating the transmitted signals into blocks with equal length to avoid the transferring of a signal error in the current block on the signal detection in other blocks. We then propose a new signal detection scheme of the MMC systems by calculating the distance between the transmitter and the receiver based on a distance prediction method and detecting signals at the receiver based on the decided adaptive concentration threshold in each time interval. To verify the efficiency of our proposed scheme, we then conducted extensive simulations by the Monte Carlo simulation, and comparisons are also made among our proposed schemes, a well-known fixed threshold signal detection scheme, the CATD scheme, the PAD scheme, and a low complexity signal detection scheme for MMC systems in terms of the BER (bit error rate). Results show that our proposed schemes can outperform these schemes regarding the BER.

\section{Introduction}

Molecular communication (MC) is a nanotechnology that uses chemical molecules as the carriers of information [1]. This makes it possible for communications to be conducted at a nano-micro scale. Due to this, MC technology can achieve communications in some special scenarios that traditional communication technology cannot work well, such as pipeline [2], human body [3], and saltwater environment $[4,5]$. With the characteristics of high efficiency and low energy consumption, MC has attracted extensive attention in many fields such as e-health [6], bioengineering [7], and environmental science [8].

According to the mobility of nanomachines (which are the basic components of an MC system), MC can be divided into two categories, i.e., fixed molecular communication (FMC) [9] and mobile molecular communication (MMC) [10]. We concentrate on the MMC in this paper. In FMC, nanomachines are deployed in fixed positions. It is generally applied in applications like health monitoring [11] and water quality monitoring [12] where nanomachines are static. In MMC, however, nanomachines can move directionally or 
randomly to perform certain tasks such as target searching [13] and drug delivering $[14,15]$. This illustrates a great potential of $\mathrm{MMC}$ in long-distance communication and cancer treatment $[16,17]$.

Available studies on MMC systems mainly concentrate on the design of modulation schemes [18-21] and signal detection schemes [22-33]. Regarding the modulation schemes, available works mainly consider FMC systems and cannot be applied to MMC systems directly. In [18], the authors proposed an isomer-based ratio shift keying (IRSK) scheme. In the scheme, multiple types of molecules with different proportions are released by the transmitter to achieve a binary or a quaternary communication. In [19], the authors proposed a modulation scheme by considering molecules of a single type. They also studied the intersymbol interference (ISI) reduction issue by adjusting the communication time. In [20], the authors proposed a concentration shift keying (CSK) scheme and a molecule shift keying (MoSK) scheme. In the CSK scheme, the same types of molecules with different numbers are detected as different signals. In the MoSK scheme, two different types of molecules with the same number are regarded as separate signals. They also investigated the channel capacity to validate the efficiency of their schemes. To obtain lower ISI, the authors in [21] proposed a depleted molecule shift keying (D-MoSK) modulation scheme by considering reduced types of molecules. They also analyzed the achievable rate and the bit error rate (BER).

Regarding the signal detection schemes for MMC systems, available works can be classified into two categories: energy-based signal detection schemes [22-25] and sampling-based signal detection schemes [26-33]. In energybased signal detection schemes, the receiver first accumulates the number of molecules absorbed by the receiver in a time period and then conducts the signal detection by comparing the calculated number with the predefined threshold of the number of molecules [34]. In these schemes, the threshold setting is a challenging work. In [22], the authors considered MC systems with a mobile transmitter and a mobile receiver communicating over an unbounded diffusive-drift channel at a variable velocity (i.e., constant speed but random direction). They proposed a signal detection scheme in which the detection threshold is set by using the maximum-likelihood estimation method. They also analyzed the BER and capacity of the system with the D-MoSK modulation scheme in [21]. To get a more accurate detection threshold, the authors in [23] considered an MC system with a pair of nanomachines communicating over an unbounded diffusive channel at variable velocities. They proposed a signal detection scheme based on the two-step maximum-likelihood method. They estimated the initial distance between the mobile nanomachines in the first step and then derived in the second step the optimal detection threshold based on the initial distance. Considering the attenuation of signal strength caused by the long communication distance between the transmitter and the receiver, the authors in [24] introduced a relay in the MC system to assist the signal transmission from the source nanomachine to the destination nanomachine over diffusive-drift channels. The scheme detects the signal by setting different detection thresholds at the relay and the destination nanomachine, respectively, and the probability of false alarm and end-to-end error was derived to validate the performance of the signal transmission in the system. To extend the work in [24], the author in [25] further investigated the ISI reduction and molecular consumption issues in MMC systems and explored the optimal number of molecules to be released at the source nanomachine and the relay, respectively.

In sampling-based detection schemes, the receiver measures the concentration of molecules at a point of time and then detects the signal by comparing the concentration with the given concentration threshold [34]. Considering only diffusive channels, the authors in [26] developed a network model of mobile cooperative tracking nanonetworks consisting multiple nanorobots of variable velocities. They proposed a signal detection scheme based on the concentration of repellents and attractants. With the scheme, the activities of nanorobots can be controlled for a target searching. To also address the problem of target searching, the authors in [27] considered an unbounded MC system with a mobile receiver moving at a variable velocity. They proposed two signal detection schemes. One scheme conducts the signal detection based on the concentration of calcium ions, and another detects the signals based on the waveform of calcium signals. In [28], the authors proposed a scheme to detect the transmitted signals as " 1 " when the concentration of alcohol molecules is above a given concentration threshold. In [29], the authors considered an MC system with a mobile full-duplex (FD) transmitter and a mobile FD receiver communicating over a channel of a limited boundary at a variable velocity. They proposed a concentration-based signal detection scheme to solve the synchronization problem by predicting the distance between the transmitter and the receiver. In [30], the authors focused on MC systems with a fixed transmitter and a mobile receiver of a variable velocity communicating over an unbounded three-dimensional (3D) diffusive channel. They proposed a concentration-based adaptive threshold detection (CATD) scheme and a peak-time-based adaptive detection (PAD) scheme by exploiting the distance estimation method which samples the concentration and calculates the average distance to reconstruct the impulse response. However, the two schemes cannot perform well when the channel characteristics change significantly. In [31], the authors considered the same MC system as [30]. They proposed a low complexity adaptive signal detection (LCSD) algorithm based on the convexity and concavity of the impulse response and the average distance is used to verify the efficiency of the scheme. However, the scheme did not consider the case of the drift issue in the channel. Considering diffusive-drift channels with a limited boundary, the authors in [32] concentrated on a 3D blood vessel digital MMC system with a transmitter of a constant velocity (i.e., constant speed and direction) and a fixed receiver. They investigated the intersymbol interference (ISI) reduction issue in the signal detection. In [33], the authors considered an MC system with a fixed transmitter communicating with 
a mobile receiver moving at a constant velocity over a $3 \mathrm{D}$ drifting diffusive channel of a limited boundary. They explored the optimal communication interval and the appropriate concentration threshold in the signal detection.

We can demonstrate from above that most modulation schemes are proposed for FMC systems, which cannot be applied to MMC systems directly as the mobility of nanomachines will introduce new problems. In addition, available signal detection schemes for MMC systems mainly consider unbounded/bounded diffusive channels and a fixed/mobile receiver or unbounded diffusive-drift channels and a mobile receiver of a variable velocity or bounded diffusive-drift channels and a mobile receiver of a constant velocity. However, schemes for MMC systems with joint consideration of the bounded diffusive-drift channel and a mobile receiver moving at a variable velocity are largely unexplored. In fact, this is a critical work to achieve a general framework for the MMC systems as follows. (1) The time for molecular diffusion will be reduced when considering the drift of communication channels. This makes it difficult to set the concentration threshold in the signal detection. (2) Molecules will reach the receiver at different times when considering a mobile receiver and a channel of a limited boundary. This will lead to the problem of bit misalignment and will result in a higher BER and thus a reduced performance of MMC systems. To address these limitations, we aim to investigate in this paper the modulation and the signal detection issues for an MMC system with a fixed transmitter communicating with a mobile receiver of a variable velocity (i.e., fixed speed but random direction) over bounded diffusive-drift channels. The contributions of the paper are illustrated as follows:

(i) We first propose a new modulation scheme to address the misalignment issue in signal detection. In the scheme, three types of molecules are adopted in the signal modulation and the transmitted signals are modulated into blocks with equal length to avoid the chain effect of a signal error in the current block on the signal detection in other blocks.

(ii) We then propose a new signal detection scheme where we first calculate the distance between the transmitter and the receiver based on a distance prediction method and then conduct the concentration threshold setting for each time interval. Finally, signals are detected at the receiver by adopting the decided adaptive concentration threshold in each time interval.

(iii) Finally, we provide extensive numerical results by the Monte Carlo simulation to verify the efficiency of our proposed schemes and to analyze the influence of different parameters on the BER. We also made a comparison among our proposed schemes, a well-known fixed threshold signal detection scheme, the CATD scheme, the PAD scheme, and a low complexity signal detection scheme. The results show that our proposed schemes can outperform these schemes in terms of the BER.
The remainder of the paper is organized as follows. Section 2 introduces the system model. Section 3 provides our new modulation scheme and signal detection scheme. Section 4 gives the case study. Section 5 provides the simulation results and discussions. Section 6 finally concludes the paper.

\section{System Model}

As illustrated in Figure 1, we consider a three-dimensional (3D) MMC system composed of a fixed transmitter and a mobile receiver continuously detecting the transmitted symbols. The transmitter and the receiver are both nanomachines communicating over a cylindrical diffusive-drift channel of an infinite length. The channel is a circular section of radius $r$. Thus, the drift is uniform and the direction of drift is from left to right horizontally. We assume that the drift velocity is $V$ and the velocity of the mobile receiver is $v$. Moreover, we consider that the receiver velocity is independent of the drift velocity in this paper, which is a common assumption in diffusive-drift molecular communication (MC) systems $[22,33]$. The transmitter is fixed at the position $\left(x_{t}, y_{t}, z_{t}\right)$ to release molecules, and the receiver with the initial position $\left(x_{0}, y_{0}, z_{0}\right)$ moves randomly in the channel to receive the instructions of the transmitter and conduct health monitoring simultaneously. The mobility model of the receiver and the communication model are provided in the following subsections.

2.1. Mobility Model of the Receiver. As the modeling of the diffusion process is still unexplored by now for our concerned system, we adopt in this paper the same mobility model of the receiver as that in [30] (the mobility model of which can be dated back to [35].). In this mobility model, the receiver is capable of moving randomly in the channel without the consideration of the complex diffusion process. The receiver in this model can work in two modes, i.e., the tumble mode and the run mode, at each time $t$. Figure 2 shows the possible activities of the receiver when it works in either mode.

The time is divided into time intervals of equal length $\Delta t$. For each $\Delta t$, the occurrence of the run mode and tumble mode follows the Poisson interval distribution with parameters $\lambda$ and $1-\lambda$, respectively [35]. Therefore, the probability that the run mode occurs between time $t$ and $t+\Delta t$ is

$$
P(t ; \lambda) \Delta t=\lambda e^{-\lambda t} \Delta t
$$

and the probability that the tumble mode occurs between time $t$ and $t+\Delta t$ is thus $1-P(t ; \lambda) \Delta t$.

In the tumble mode, the receiver can change its moving direction (e.g., from $\theta(t)$ at the time $t$ to $\theta(t+\Delta t)$ at the time $t+\Delta t$ in Figure 2). In $3 \mathrm{D}$ environments, the moving direction of the receiver can be modeled by a three-dimensional angle $\theta(t)=\left(\theta_{x y}(t), \theta_{z}(t)\right)$ [30]. Here, $\theta_{x y}(\cdot)$ is the azimuthal angle and $\theta_{z}(\cdot)$ is the polar angle, and the angle $\theta_{x y}$ $(t+\Delta t)$ and $\theta_{z}(t+\Delta t)$ at time $t+\Delta t$ can be given by 


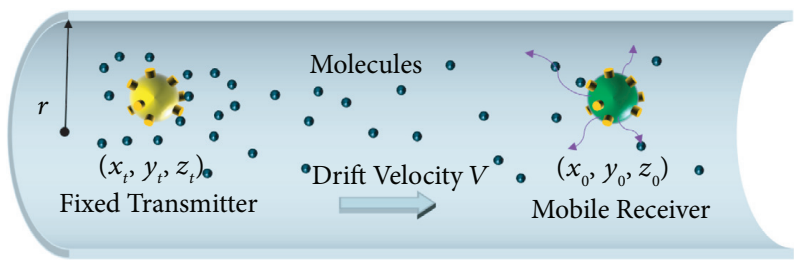

FIGURE 1: Illustration of the system model.

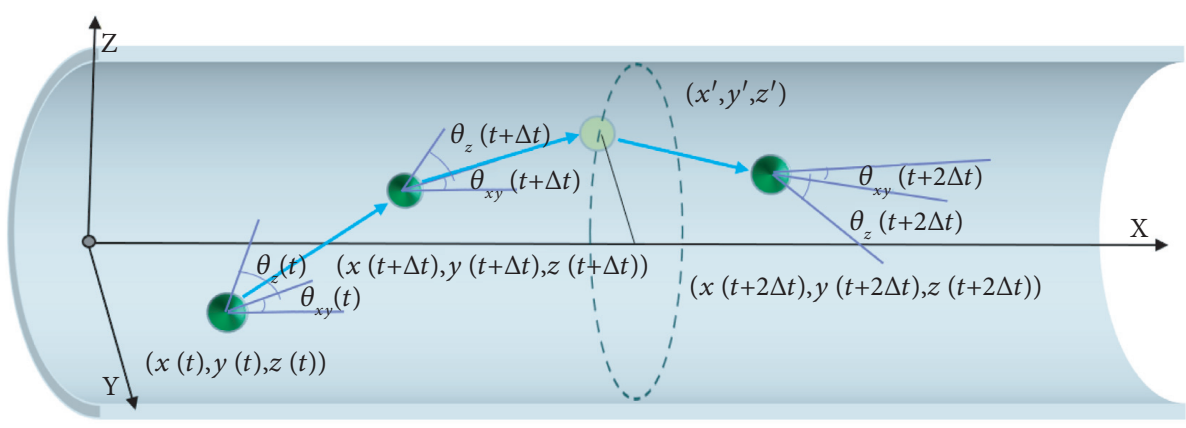

Figure 2: Possible activities of the receiver.

$$
\begin{gathered}
\theta_{x y}(t+\Delta t)=\theta_{x y}(t)+\Phi \\
\theta_{z}(t+\Delta t)=\theta_{z}(t)+\Phi
\end{gathered}
$$

where $\Phi=+\phi, \phi$ denotes the rotation angle of the receiver, and $\phi$ is determined as [26]

$$
\phi=\left|\sqrt{2 D_{r} \Delta t}\right|
$$

where $D_{r}$ is the rotational diffusion coefficient indicating the rotating ability of the receiver. Please notice that a larger $D_{r}$ can make sure that the receiver can turn around in a small range, but the receiver can move in just one direction under a lower $D_{r}$ case. Assuming that $\theta(0)$ is the initial moving direction of the receiver, it has been proved in [26] that

$$
E[\theta(t)]=\theta(0),
$$

where $E[\cdot]$ is the expectation operator.

In the run mode, the receiver can run toward its moving direction. Due to the assumption of limited radius $r$ of the channel, two possible cases may occur during the running of the receiver, i.e., running without boundary collision and running with boundary collision. For the receivers running without boundary collision, it moves within the channel. For example, when it runs from the position $(x(t), y(t), z(t))$ at time $t$ to the position $(x(t+\Delta t), y(t+\Delta t), z(t+\Delta t))$ at time $t+\Delta t$ with three-dimensional angle $\theta(t)$ in Figure 2, then we have

$$
\begin{aligned}
& x(t+\Delta t)=x(t)+v \Delta t \sin \theta_{z}(t) \cos \theta_{x y}(t), \\
& y(t+\Delta t)=y(t)+v \Delta t \sin \theta_{z}(t) \sin \theta_{x y}(t), \\
& z(t+\Delta t)=z(t)+v \Delta t \cos \theta_{z}(t)
\end{aligned}
$$

where $v$ is the velocity that the receiver moves in the channel (i.e., moving velocity).
For the receiver's running with boundary collision, it moves near the boundary of the channel and has a collision with the boundary [26]. See the running of the receiver from the position $(x(t+\Delta t), y(t+\Delta t)$, and $z(t+\Delta t))$ to the position $(x(t+2 \Delta t), y(t+2 \Delta t)$, and $z(t+2 \Delta t))$ in Figure 2 where there is a collision with the boundary of the channel at the position $\left(x^{\prime}, y^{\prime}, z^{\prime}\right)$. We set the radius at which the collision point is connected to the center of the circular cross section as $r_{x^{\prime}}$. In $3 \mathrm{D}$ space, the mobile receiver's trajectory after a collision can be obtained by symmetrically combining the trajectory before a collision with the radius $r_{x^{\prime}}$. Therefore, if the receiver moves at the position $(x(t), y(t), z(t))$ at time $t$, then the distance between the receiver and the transmitter can be expressed as [30]

$$
d(t)=\sqrt{\left[x(t)-x_{t}\right]^{2}+\left[y(t)-y_{t}\right]^{2}+\left[z(t)-z_{t}\right]^{2}}
$$

where $x_{t}, y_{t}$, and $z_{t}$ are obtained from the position of the transmitter.

2.2. Communication Model. Figure 3 shows the communication model of the MMC system. For a sequence of symbols $a_{1}, a_{2}, \ldots, a_{n}, \ldots, a_{N}$ to be transmitted from the transmitter to the receiver over a diffusive-drift channel as that in [33], the transmitter first modulates the symbols into molecule concentration pulse $s(t)$ by adopting the modulation schemes. For example, we use the on-off keying (OOK) modulation scheme where the symbol sequence is modulated by releasing $M$ molecules to represent the bit " 1 ," and sending 0 molecules to represent the bit " 0 ." Assuming that the time cost for the molecule releasing $\delta$ is extremely short [30], then the molecule concentration pulse can be expressed as 


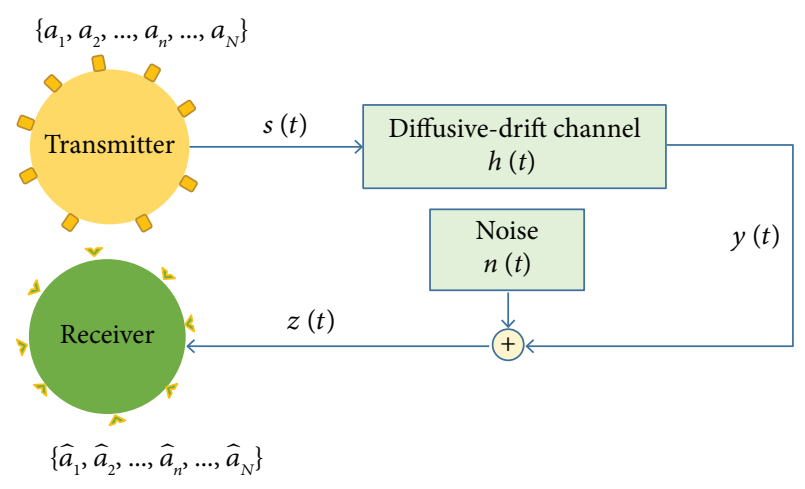

Figure 3: The communication model of the MMC system with a diffusive-drift channel.

$$
s(t) \approx \sum_{n=1}^{\infty} a_{n} M \delta(t-(n-1) T)
$$

where $T$ is the transmission interval between the last communication and the current communication, $M$ is the number of molecules released in the channel, and $\delta(t-(n-$ 1)T) is the molecular releasing duration.

After the modulation, the transmitter releases molecules to the channel which has an impulse response $h(t)$. As the molecules will diffuse at infinite dilution according to the channel model in [33], the impulse response is achieved as

$$
h(t)=\frac{1}{(4 \pi D t)^{3 / 2}} \exp \left[\frac{(d(t)-V t)^{2}}{4 D t}\right],
$$

where $D$ is the diffusion coefficient, which indicates the diffusion ability of molecules, $d(t)$ is given in (6), and $V$ is the drift velocity, and the total concentration detected by the receiver is given by

$$
y(t)=s(t) * h(t)=\sum_{n=1}^{\infty} a_{n} C(t-(n-1) T),
$$

where * denotes the convolution operator, $C(t)$ is a function representing the detected concentration by the receiver at the time $t, h(t)$ (which is given in (8)) is the impulse response, and we have

$$
C(t)=M h(t)=\frac{M}{(4 \pi D t)^{3 / 2}} \exp \left[-\frac{(d(t)-V t)^{2}}{4 D t}\right] .
$$

In addition, as the molecules can randomly move in the channel, counting noise will be introduced in the system. Considering the Additive White Gaussian Noise (AWGN) in the channel, we then have the counting noise $n(t)$ as

$$
n(t) \sim N\left[0, \sigma_{c}^{2}(t)\right]
$$

where $\sigma_{c}^{2}(t)$ denotes the variance and

$$
\sigma_{c}^{2}(t)=\frac{3 y(t)}{4 \pi \rho^{3}}
$$

where $\rho$ is the radius of the receiver.

Combining (9) and (11), we then can obtain the concentration detected by the receiver as

$$
z(t)=y(t)+n(t)=\sum_{n=1}^{\infty} a_{n} C(t-(n-1) T)+n(t)
$$

With $z(t)$, the receiver can finally conduct the demodulation and obtain a symbol sequence $\widehat{a}_{1}, \widehat{a}_{2}, \ldots, \widehat{a}_{n}, \ldots, \widehat{a}_{N}$ in terms of the symbol sequence $a_{1}, a_{2}, \ldots, a_{n}, \ldots, a_{N}$ by using a demodulation scheme.

\section{Proposed Schemes}

In this section, we propose a new modulation scheme and a new signal detection scheme for MMC systems with a fixed transmitter communicating with a mobile receiver over a diffusive-drift channel.

3.1. Modulation Scheme. Available modulation schemes are mainly applicable for FMC systems, which cannot be applied to MMC systems directly. The modulation schemes for FMC systems will cause the misalignment in the signal detection of an MMC system. Motivated by the MoSK scheme, we propose a new modulation scheme by applying three types of molecules (i.e., molecule A, molecule B, and molecule $C$ ) in this paper. Here, the molecules $A$ and $B$ are used to modulate the symbol " 1 " and " 0 ", respectively, and molecule $C$ is used to block the information. The idea of using a start molecule $\mathrm{C}$ is innovative in the context of the chunked-transferencoding mechanism, where extra bits are allocated at the beginning of each information block before the information encoding to indicate the ends/start of a block transmission [36]. Assuming a block of length $L$, a sequence of symbols to be transmitted at the transmitter are modulated according to the following rule:

(i) Divide symbols in the sequence into blocks of equal length $L$

(ii) Use the molecule $\mathrm{C}$ as the beginning of each information block

(iii) Modulate the symbol " 1 " (“0") in each block by releasing molecule $\mathrm{A}(\mathrm{B})$

Table 1 shows an example of the proposed modulation scheme when transmitting a sequence of symbols consisting of 6 bits of " 100110 " at the transmitter and the block length is set as $L=3$. According to the modulation rule, "100110" will be modulated into two blocks "CABB" and "CAAB."

From the modulation rule, we can see that, with the introduction of a third molecule $\mathrm{C}$ in the modulation process, our proposed modulation scheme can keep the misalignment in one block and the misalignment will not be transferred to the following blocks by modulating the transmitted symbols into several blocks. This will greatly reduce the probability of misalignment in the signal detection of an MMC system.

3.2. Signal Detection Scheme. Due to the mobility of nanomachines, a higher BER is introduced in available signal detection schemes for MMC systems with a fixed concentration threshold. To reduce the BER of MMC systems, we 
TABLE 1: An example of our proposed modulation scheme.

\begin{tabular}{|c|c|c|c|c|c|c|c|c|}
\hline Bits symbols & & 1 & 0 & 0 & & 1 & 1 & $\overline{0}$ \\
\hline Molecule & $\mathrm{C}$ & $\mathrm{A}$ & B & B & $\mathrm{C}$ & A & A & B \\
\hline
\end{tabular}

propose a new signal detection scheme by adopting a dynamic molecule concentration threshold in this subsection where we first give the method of dynamic threshold setting and then provide our new signal detection scheme with the dynamic threshold.

3.2.1. Concentration Threshold Setting. Different from available works with fixed concentration thresholds, we adopt a dynamic concentration threshold for the whole transmission of a symbol sequence in this paper. The basic idea of our threshold setting method includes three steps. In the first step, we calculate the actual distance between the transmitter and the receiver for each block when molecule $\mathrm{C}$ reaches its peak concentration based on its peak concentration and peak time. In the second step, we will predict the maximum distance between the transmitter and the receiver in each of the following time intervals of the whole transmission of a symbol sequence based on the calculated actual distance of each block and the speed of the receiver. In the third step, the concentration threshold for each time interval is obtained with the help of the calculated actual distance and the predicted maximum distance between the transmitter and the receiver. Details of the three steps are shown as follows.

Step 1: Actual Distance Calculation. The first step in setting the concentration threshold is to estimate the current actual communication distance. Available works on distance estimation can be mainly divided into two categories: one is the round-trip-based distance estimation schemes [37, 38], and another is the one-way-based distance estimation schemes [31, 39-42]. In the round-trip-based distance estimation schemes, the distance between the transmitter and the receiver is estimated based on the feedback of the receiver, which is time-consuming and difficult to be deployed in a communication environment with drift. In the one-way distance estimation schemes, however, the distance between the transmitter and the receiver is estimated based on the sampled concentration (e.g., the maximum-likelihood (ML) method $[39,40]$ and peak detection method [41, 42].) In this paper, we use the peak detection method in [41] for the actual distance calculation.

Adopting the modulation scheme in Section 3.1, the transmitter releases molecule $\mathrm{C}$ at the beginning of each block (e.g., the $i_{\text {th }}$ block), and the residual of molecule $C$ in the channel can be ignored as the block length $L$ goes to be long enough (see Lemma 1 in the appendix for the proof). Denoting the peak concentration of molecule $\mathrm{C}$ detected by the receiver as $\delta_{c, i}^{\text {peak }}$, the actual distance between the transmitter and the receiver for the $i_{\text {th }}$ block at the peak time $\delta_{c, i}^{\text {peak }}$ of molecule C can be obtained based on (10) as

$$
d_{0}^{i}=d\left(t_{c, i}^{\text {peak }}\right)=\left\{-4 D Q_{i}^{\text {peak }} \ln \left[\frac{\delta_{c, i}^{\text {peak }}}{M}\left(4 \pi D Q_{i}^{\text {peak }}\right)^{3 / 2}\right]\right\}^{1 / 2}+V Q_{i}^{\text {peak }}, \quad i=1,2, \ldots
$$

where $D$ is the diffusion coefficient in (8), $M$ is the number of released molecules, $V$ is the drift speed of the channel, and $Q_{i}^{\text {peak }}$ is the time it takes for the molecule $C$ to reach its peak concentration for the $i_{\text {th }}$ block, $Q_{i}^{\text {peak }}=t_{c, i}^{\text {peak }}-(i-1) L T$, $\delta_{c, i}^{\text {peak }}=C\left(Q_{i}^{\text {peak }}\right)$, and $C\left(Q_{i}^{\text {peak }}\right)$ is given in (10) by setting $t=Q_{i}^{C\left(Q_{i}^{\text {peak }}\right)}$.

Step 2: Maximum Distance Prediction. As we consider a mobile receiver that moves randomly in the channel of MMC systems, the receiver can move toward any direction with any rotation angle. This makes it difficult for the threshold setting in MMC systems. To address this issue, we predict in this step the maximum distance between the transmitter and the receiver in the following intervals of each block after the actual distance calculation. We consider an extreme case that the receiver moves directly away from the transmitter at a constant speed $v$ after the molecule $\mathrm{C}$ reaches its peak concentration and consider no rotation of the receiver.

For the $i_{\text {th }}$ block, the number of time intervals during which the molecule $C$ reaches its peak concentration can be obtained as $t_{c, i}^{\text {peak }} / T$, where $t_{c, i}^{\text {peak }}$ is the peak time of molecule C. Let $j=0,1,2,3, \ldots$; the maximum predicted distance between the transmitter and the receiver for the $\left(t_{c, i}^{\text {peak }} / T+j\right)_{\text {th }}$ time interval $T$ is determined as

$$
d_{p}\left(t_{c, i}^{\text {peak }}+j T\right)= \begin{cases}d_{0}^{i}, & j=0, \\ d_{p}\left(t_{c, i}^{\text {peak }}+(j-1) T\right)+v T, & j=1,2, \ldots,\end{cases}
$$

where $d_{p}(\cdot)$ denotes the maximum distance prediction function, $T$ is the time interval, $v$ is the velocity of the mobile receiver, $t_{c, i}^{\text {peak }}$ is the time at which molecule $C$ reaches its peak concentration, and $d_{0}^{i}$ (which is given in (14)) is the actual distance between the source and the receiver.

Step 3: Concentration Threshold Setting. With the predicted maximum distance, the concentration threshold for the $\left(t_{c, i}^{\mathrm{peak}} / T+j\right)_{\text {th }}$ time interval $T$ is set by first calculating the time it costs the molecule to reach its peak concentration after it is released by the transmitter and then setting the value of the concentration threshold as the peak concentration of the molecule.

Based on formula (10), the peak time at which the molecule reaches its peak concentration in the $\left(t_{c, i}^{\text {peak }} / T+j\right)_{\text {th }}$ time interval can be achieved as 


$$
t_{t_{c, i}^{\text {peak }} / T+j}^{\text {peak }}-\frac{\sqrt{9 D^{2}+V^{2} d_{p}\left(t_{c, i}^{\text {peak }}+j T\right)^{2}}-3 D}{V^{2}}+(i-1) L T,
$$

by substituting the predicted maximum distance in (15) into (10) and then conducting the derivation.

With the peak time $t_{c, i}^{\text {peak }} / T+j$, the time it costs the molecule in the $\left(t_{c, i}^{\text {peak }} / T+j\right)_{\text {th }}$ time interval to reach its peak concentration after being released at the transmitter can be obtained as

$$
Q_{t_{c, i}^{\text {peak }} / T+j}^{\text {peak }}=\frac{\sqrt{9 D^{2}+V^{2} d_{p}\left(t_{c, i}^{\text {peak }}+j T\right)^{2}}-3 D}{V^{2}} .
$$

Combining the results in (16) and (17), the concentration threshold for the $\left(t_{c, i}^{\text {peak }} / T+j\right)_{\text {th }}$ time interval is finally set as

$$
\begin{aligned}
\tau_{t_{c, i}^{\text {peak }} / T+j}= & \left.C\left(Q_{t_{c, i}^{\text {peak }} / T+j}^{\text {peak }}\right)=\frac{M}{\left(4 \pi D Q_{t_{c, i}^{\text {peak }} / T+j}^{\text {peak }}\right.}\right)^{3 / 2} \\
& \cdot \exp \left[\frac{-\left(\left(d_{p}\left(t_{c, i}^{\text {peak }}+j T\right)+j T\right)-V Q_{t_{c, i}^{\text {peak }} / T+j}^{\text {peak }}\right)^{2}}{4 D Q_{t_{c, i}^{\text {peak }} / T+j}^{\text {peak }}}\right] .
\end{aligned}
$$

Specially, when $v=0$ (i.e., the receiver is fixed), equation (18) can be simplified as

$$
\begin{aligned}
\tau_{t_{c, i}^{\text {peak }} / T+j}=C\left(Q_{t_{c, i}^{\text {peak }} / T+j}^{\text {peak }}\right)= & \left.\frac{M}{\left(4 \pi D Q_{t_{c, i}^{\text {peak }} / T+j}^{\text {peak }}\right.}\right)^{3 / 2} \\
& \cdot \exp \left[\frac{-\left(d_{0}^{i}-V Q_{t_{c, i}^{\text {peak }} / T+j}^{\text {peak }}\right)^{2}}{4 D Q_{t_{c, i}^{\text {peak }} / T+j}^{\text {peak }}}\right],
\end{aligned}
$$

where $d_{0}^{i}$, which is given in (14), is the actual distance between the source and the receiver.

3.2.2. Signal Detection. Based on the system model in Section 2, the receiver is continuously detecting the concentration of transmitted molecules during the whole transmission of a symbol sequence. Traditional signal detection methods detect the signals as " 1 " or " 0 " when the concentration of molecules detected by the receiver is above the concentration threshold in a time interval. However, these methods cannot be applied to situations where the peak concentration appears more than once in a time interval. To address this issue, we propose the following new signal detection method in this paper.
Assuming that a bit " 1 " is transmitted from the transmitter, then it will be modulated as molecule A according to our modulation scheme in Section 3.1. We denote $T_{s}$ as the sampling interval of the receiver (i.e., signal detection is conducted once after each time interval $T_{s}$ ) and denote $\tau_{k}^{A}$ (which is derived according to the concentration threshold setting method in (18)) as the concentration threshold of molecule $\mathrm{A}$ in the $k_{\mathrm{th}}$ time interval; then, molecule $\mathrm{A}$ is correctly detected as the bit " 1 " if and only if there exist the times $t_{1}$ and $t_{2}\left(t_{1}<t_{2}\right)$ such that

$$
\begin{aligned}
& \left(\widehat{Z}^{A}\left(t_{1}\right) \leq \tau_{k}^{A}\right) \cap\left(\widehat{Z}^{A}\left(t_{1}+T_{s}\right) \geq \tau_{k}^{A}\right), \\
& \left(\widehat{Z}^{A}\left(t_{2}\right) \leq \tau_{k}^{A}\right) \cap\left(\widehat{Z}^{A}\left(t_{2}+T_{s}\right) \geq \tau_{k}^{A}\right),
\end{aligned}
$$

where $\widehat{Z}^{A}(\cdot)$ is the concentration of molecule A after the mitigation $[33,43]$ and

$$
\widehat{Z}^{A}\left(t_{i}\right)=Z^{A}\left(t_{i}\right)-Z_{I S I}^{A}\left(t_{i}\right)
$$

, where $Z^{A}(\cdot)$ is the detected concentration by the receiver and $Z_{I S I}^{A}(\cdot)$ is the residual concentration of molecule $A$ when the $n_{\text {th }}$ symbol sequence of molecular A $\left\{a_{1}^{A}, a_{2}^{A}, \cdots, a_{n}^{A}, \cdots\right\}$ is detected by the receiver. Here, we define $a_{n}^{A}=1\left(a_{n}^{A}=0\right)$ when $A$ is (is not) released and

$$
Z_{I S I}^{A}\left(t_{i}\right)=\sum_{j=1}^{n-1} a_{j}^{A} C^{A}\left(t_{i}-(j-1) T\right), \quad i \in\{1,2\},
$$

where $C^{A}\left(t_{i}-(j-1) T\right)$ is given by

$$
\begin{aligned}
C^{A}\left(t_{i}-(j-1) T\right)= & \frac{M^{A}}{\left[4 \pi D\left(t_{i}-(j-1) T\right)\right]^{3 / 2}} \\
& \times \exp \left[\frac{-\left(d_{p}\left(t_{i}\right)-V\left(t_{i}-(j-1) T\right)\right)^{2}}{4 D\left(t_{i}-(j-1) T\right)}\right],
\end{aligned}
$$

where $M^{A}$ is the number of molecule A released by the transmitter, $D$ is the diffusion coefficient, and $d_{p}\left(t_{i}\right)$, which is given in (15), is the predicted maximum distance between the transmitter and the receiver at time $t_{i}$.

We can observe from our signal detection method that any molecule that reaches its peak concentration in the time interval between $t_{1}$ and $t_{2}$ can be detected correctly, which protects molecules from misdetection. Therefore, our signal detection scheme can detect the signals correctly even if the peak concentration appears more than once in a time interval.

It is also worth noting that our detection scheme can also be applied to the signal detection of other molecules (e.g., molecules $\mathrm{B}$ and $\mathrm{C}$ ).

\section{Case Study}

To illustrate the efficiency of our proposed schemes, this section provides a case study for communications in MMC 
systems with our new modulation scheme, signal detection scheme, and the conventional MoSK modulation scheme.

Considering a symbol sequence of " 100110 " to be transmitted at the transmitter, in the MoSK scheme, the symbol sequence " 100110 " is modulated as a whole block "ABBAAB" by adopting two types of molecules (i.e., molecule A and molecule B), where the symbol " 1 " is modulated as molecule A and the symbol " 0 " is modulated as molecule B. In our proposed modulation scheme, however, three types of molecules (i.e., molecule A, molecule B, and molecule C) are applied and the symbol sequence " 100110 " can be modulated into several blocks of equal length. Considering the block length of 3 , the symbol sequence " 100110 " is modulated as "CABB" and "CAAB" according to our modulation scheme in Section 3.1. For the signal detection process, a fixed concentration threshold is used in the MoSK scheme, and a molecule is detected by the receiver in a time interval if and only if the detected concentration at the receiver is not lower than the given threshold. In our new signal detection schemes, an adaptive threshold is adopted and the receiver detects a molecule when meeting the conditions in (20) and (21).

Figures 4(a) and 4(b) show the results of molecular concentration in different time intervals for the MMC systems in Section 2 with the MoSK scheme and our proposed modulation and signal detection schemes, respectively. It can be observed from Figure 4(a) that misalignment may be introduced in signal detection. For example, molecules detected in the time interval $4 T$ to $5 T$ should be detected during the time interval from $3 T$ to $4 T$. This will cause a detection error of the $4_{\text {th }}$ symbol and will lead to error detection of the following symbols (i.e., the $5_{\text {th }}$ and the $6_{\text {th }}$ symbols). In addition, molecules may be undetected by the receiver (e.g., molecules transmitted from the time interval $4 T$ to $7 T$ ) when the concentration of the molecule detected by the receiver is lower than the given threshold.

By adopting our proposed modulation and signal detection schemes, we can see from Figure 4(b) that molecules can always be detected by the receiver as we set the concentration threshold in each time interval adaptively based on the predicted distance between the transmitter and the receiver. It is worth noting that even if some molecules are undetected by the receiver, it will affect only the signal detection of the following molecules in the current block and cause no effect on the signal detection in the subsequent blocks as we will use molecule $C$ to mark the start of a new block where a new subsequence of symbols will be transmitted. Moreover, although there is also misalignment during the transmission (e.g., molecules detected during the time intervals from $4 T$ to $9 T$ ), the misalignment has no impact on the detection of each symbol. This is due to the reason that the receiver detects molecules when their concentrations meet the conditions in (20) and (21), with which molecules can be detected in any time interval instead of being detected in a fixed time interval as in the MoSK scheme. Thus, our proposed modulation and signal detection schemes can solve the problems of misalignment and greatly reduce the probability of signal undetection in MMC systems.

\section{Numerical Results and Discussions}

In this section, we first provide simulation results to verify the efficiency of our proposed schemes in terms of the BER and then explored how the system parameters (e.g., the block length, the drift velocity of channel media, the number of released molecules, the velocity of the receiver, and the rotational diffusion coefficient of the receiver) will affect the BER performance of our proposed schemes. Finally, a comparison is made among our proposed schemes and a well-known fixed threshold signal detection scheme, the CATD scheme, the PAD scheme, and the LCSD scheme regarding the $\mathrm{BER}$.

5.1. Simulation Settings. We performed extensive simulations using a simulator developed based on Monte Carlo simulation, which is now available at [44]. In the simulation, a nanomachine works as the fixed transmitter and a flagellated bacterium of random walk acts as the mobile receiver $[30,45,46]$. We assume that there is a drift velocity in the environment and molecules released by the transmitter undergo the Brownian movement. Table 2 illustrates the settings of default parameters in the simulation [30, 33, 47]. At the beginning of each time interval $T$, the transmitter modulates the transmitted symbols into molecules based on the modulation scheme in Section 3.1. After the modulation, the transmitter releases molecules into the channel, and the molecules move randomly in the diffusive-drift channel. Based on the signal detection scheme in Section 3.2, the receiver finally receives the symbols by detecting the concentration of each molecule.

Due to the impacts of the mobile receiver and the drift channel, transmitted symbols (measured in bits) may be incorrectly detected at the receiver, resulting in error bits at the receiver. Denoting $N_{e}$ as the number of error bits received at the receiver and $N_{t}$ as the total number of bits transmitted at the transmitter, the simulated BER of the considered MMC system is given as

$$
\operatorname{BER}=\frac{N_{e}}{N_{t}} .
$$

5.2. Performance Discussion. With the help of our theoretical analysis, we now explore how the system parameters (i.e., the sequence length $N$, the block length $L$, the drift velocity $V$, the number of released molecules $M$, the moving velocity of the receiver $v$, and the rotational diffusion coefficient $D_{r}$ ) will affect the BER.

By setting the sequence length from 1000 to 7000 bits, we first explore in Figures 59 how the sequence length $N$ will affect the BER. It can be observed from Figures 5 to 9 that the BER increases as the sequence length $N$ increases. According to the modulation and signal detection schemes in Section 3, the number of blocks increases as the sequence length $N$ increases for a given block length. A bit is detected as an error bit if and only if there is a signal error during the communication. More blocks mean a higher ISI and thus a higher probability of signal detection errors during the 


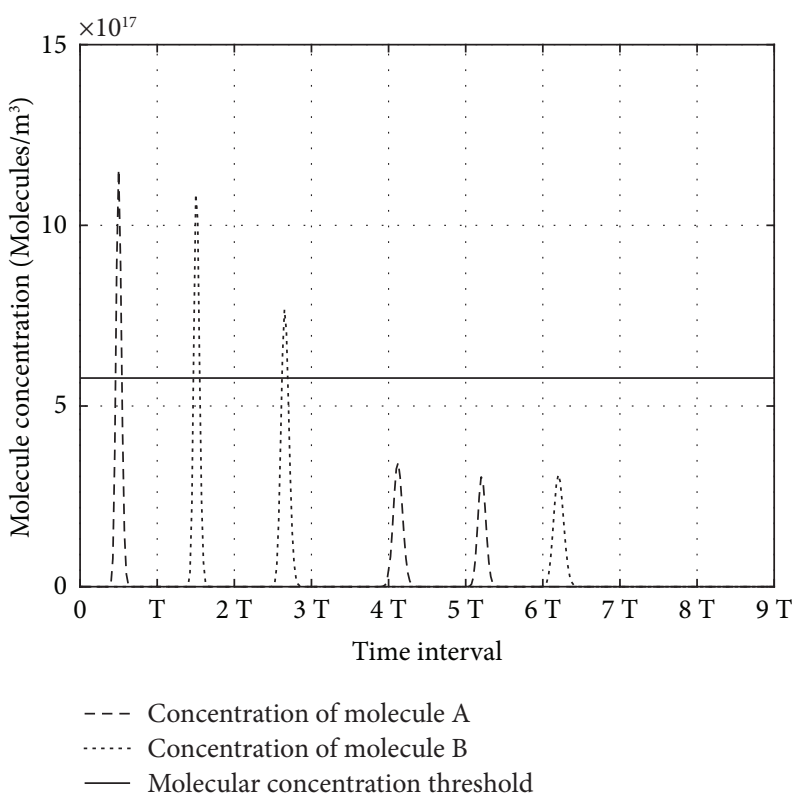

(a)

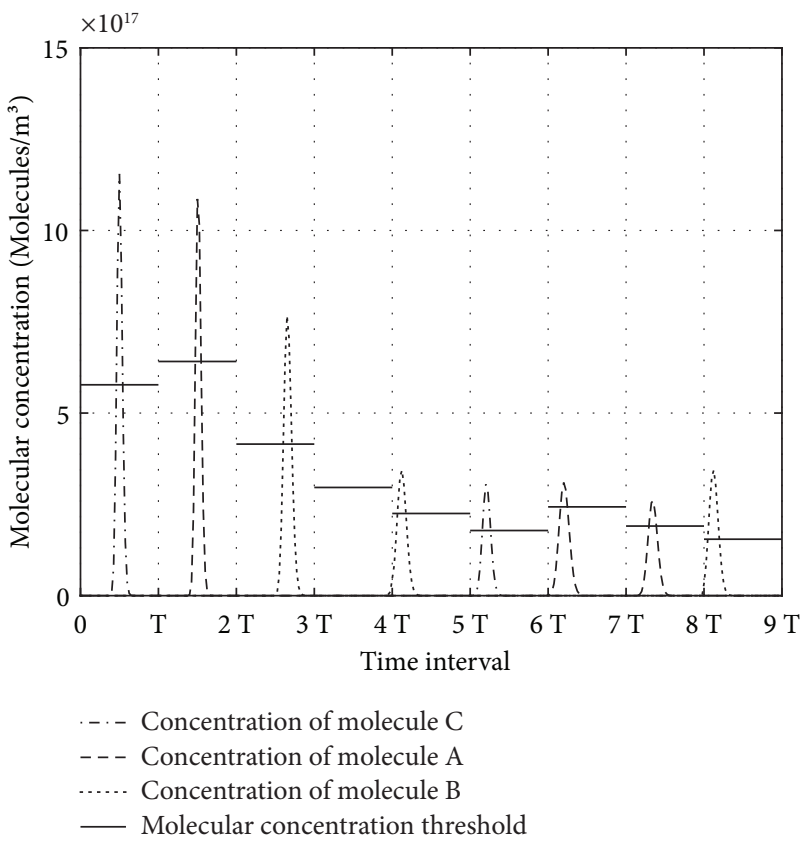

(b)

Figure 4: A case study for a symbol sequence "100110." (a) Molecular concentration in different time intervals for MMC systems in Section 2 with the MoSK modulation scheme. (b) Molecular concentration in different time intervals for MMC systems in Section 2 with our proposed modulation and signal detection schemes.

TABLE 2: Simulation parameters.

\begin{tabular}{lcc}
\hline Parameters & Symbol & Value \\
\hline Channel radius & $r$ & $30 \times 10^{-6} \mathrm{~m}$ \\
Radius of the receiver & $\rho$ & $10^{-6} \mathrm{~m}$ \\
Initial distance & $d_{0}$ & $5 \times 10^{-4} \mathrm{~m}$ \\
Drift velocity & $V$ & $5 \times 10^{-4} \mathrm{~m} / \mathrm{s}$ \\
Velocity of the receiver & $v$ & $1.975 \times 10^{-4} \mathrm{~m} / \mathrm{s}$ \\
Diffusion coefficient & $D$ & $10^{-9} \mathrm{~m}^{2} / \mathrm{s}$ \\
Rotational diffusion coefficient & $D_{r}$ & $5 \mathrm{rad}^{2} / \mathrm{s}$ \\
Number of released molecules & $M$ & $3 \times 10^{6}$ \\
Occurrence parameter of run modes & $\lambda$ & 0.9 \\
Time interval & $T$ & $2 \mathrm{~s}$ \\
Sampling step length & $\mathrm{T}_{s}$ & $2 \mathrm{~ms}$ \\
Block length & $L$ & 9 \\
\hline
\end{tabular}

communication. This will cause a higher BER of the system. Thus, the BER increases as the sequence length $N$ increases.

Next, we investigate the impacts of block length $L$ on the BER for given $N, V, v, D_{r}$, and $M$ in Figure 5. From Figure 5, we can see that the BER increases as $L$ increases. According to the new modulation scheme in Section 3.1, each symbol sequence to be transmitted in the system is divided into blocks of equal length $L$. When $L$ increases, the number of transmitted bits included in the block increases. An occurrence of any signal error in each block may lead to signal errors for the subsequent signals in the block according to the signal detection rule. Since more signal errors represent a higher BER of the system, the BER thus increases as the block length increases. Although lower values of $L$ can improve the BER performance of the MMC systems, it will add more parasitic symbols $C$ to the sequence and thus will reduce the channel capacity of the MMC system. However, studying the channel capacity is challenging [48, 49], and we will not consider the capacity issue in this paper.

We then explore in Figure 6 how the drift velocity $V$ will affect the BER of the concerned MMC system with our proposed schemes by setting various values of the drift velocity $V$. Results in Figure 6 indicate that our proposed scheme can provide a lower BER performance for higher drift velocities. This is because there will be less molecule residue when using a higher drift velocity, which will introduce less signal interference during the signal detection and thus will lead to a lower BER. Moreover, with a higher drift velocity $V$, the concentration detected by the receiver increases/decreases rapidly, and the peak concentration of a molecule is more obvious, which will benefit the signal detection at the receiver. Thus, the BER of the system 


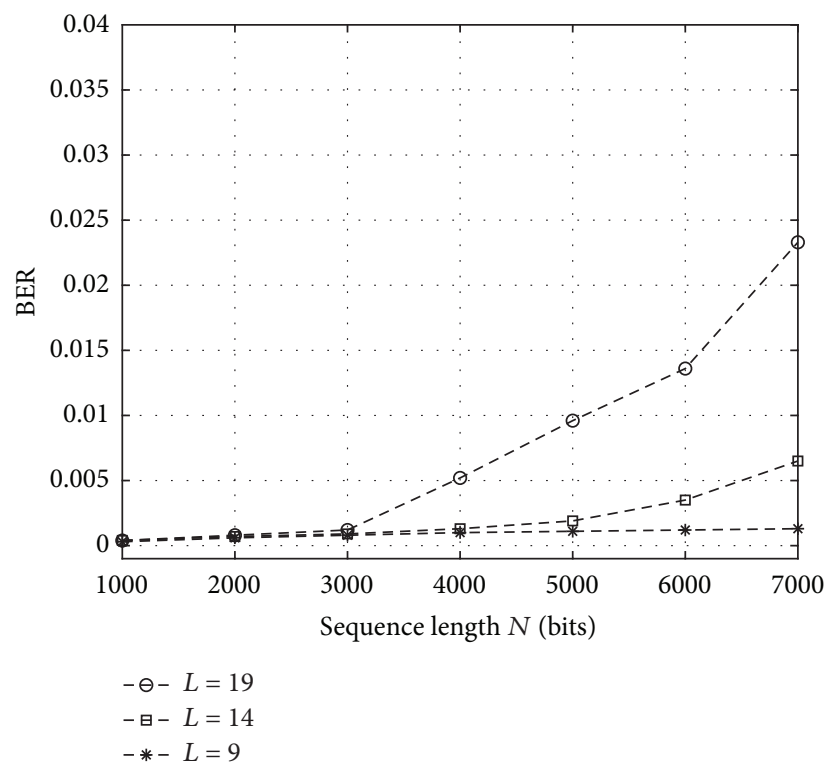

Figure 5: BER vs. the sequence length $N$ for different block lengths $L$.

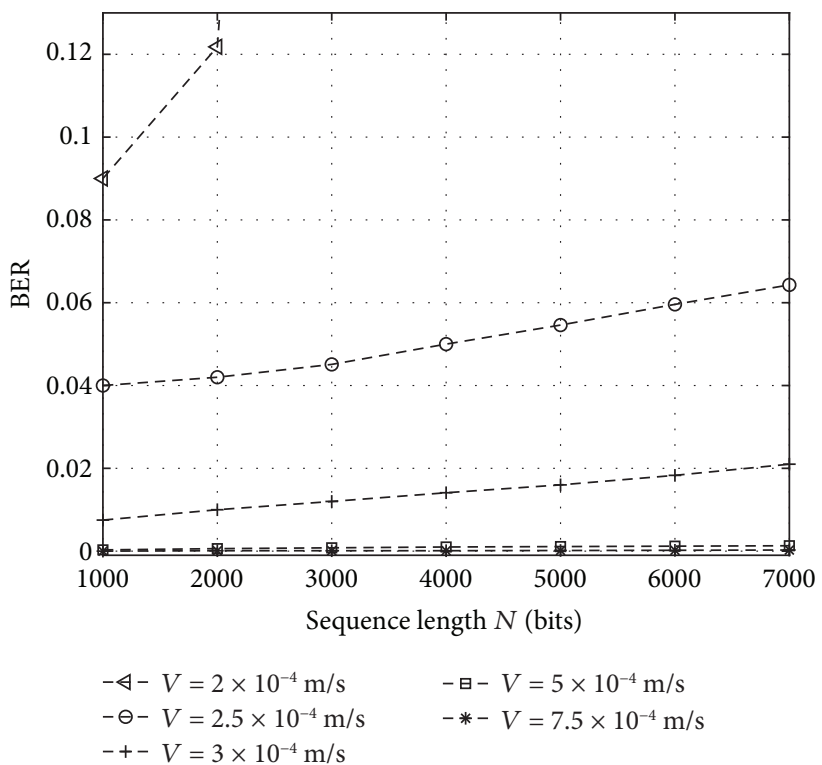

FIgURE 6: BER vs. the sequence length $N$ for different drift velocities $V$.

decreases as the drift velocity increases. We can further observe from Figure 6 that the BER increases rapidly as the drift velocity $V$ decreases below a certain value (e.g., $V=2.5 \times 10^{-4} \mathrm{~m} / \mathrm{s}$ in Figure 6). This is because there are more residual information molecules in the channel for a lower drift velocity, which will introduce much more ISI and thus will lead to a higher BER. Therefore, our scheme is suitable for MMC systems with higher drift velocities. With this observation, a minimum drift velocity can be achieved to ensure a better BER performance of the concerned system for various system settings (e.g., $V=2.5 \times 10^{-4} \mathrm{~m} / \mathrm{s}$ for the system settings of $v=1.975 \times 0^{-4} \mathrm{~m} / \mathrm{s}, \quad T=2 \mathrm{~s}$ and $\left.D_{r}=5 \mathrm{rad}^{2} / \mathrm{s}\right)$.

We examine in Figure 7 how the BER changes by setting the number of released molecules from $3 \times 10^{5}$ to $3 \times 10^{7}$. We can see from Figure 7 that the BER decreases as the number of molecules $M$ increases. Since signals are detected at the receiver based on the concentration of molecules according to the signal detection scheme in Section 3.2, much more released molecules at the transmitter mean a higher accuracy of the signal detection at the receiver. Thus, the BER decreases as the number of molecules $M$ increases. 


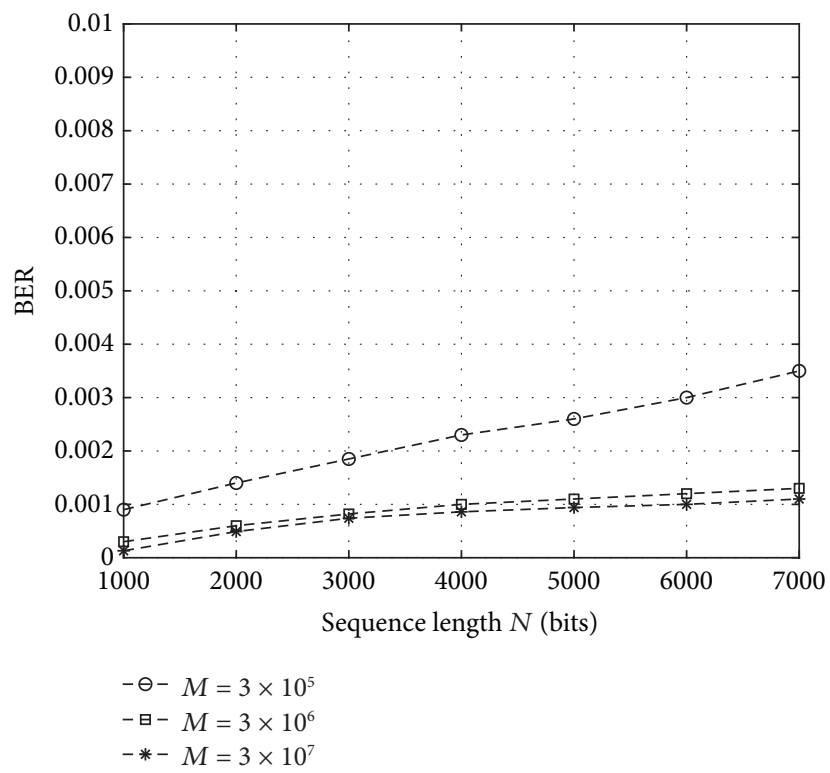

FIgURE 7: BER vs. the sequence length $N$ for different numbers of released molecules $M$.

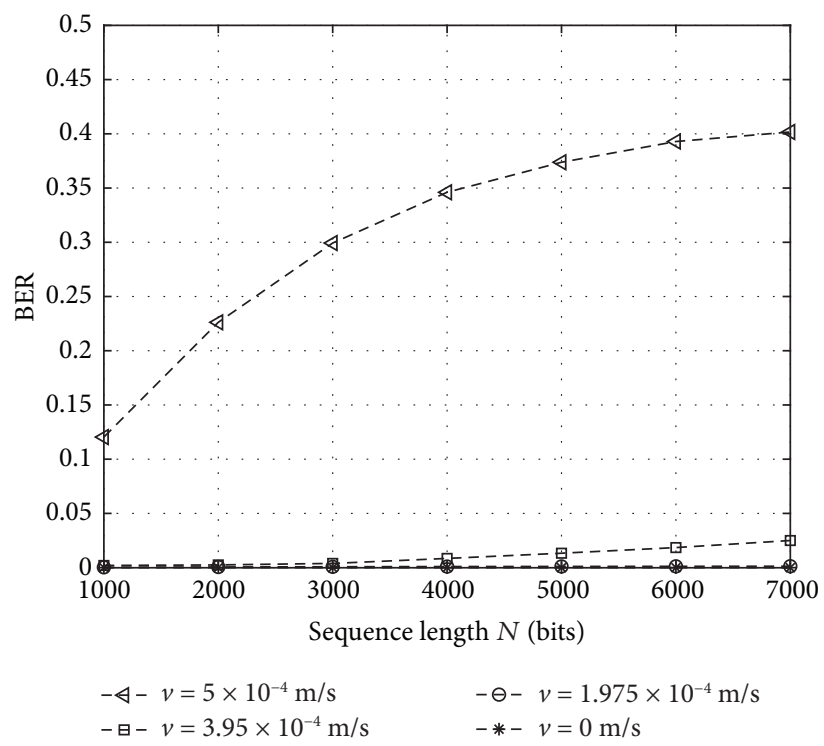

FIgURE 8: BER vs. the sequence length $N$ for different moving velocities of the receiver $v$.

We then explore how the moving velocity of receiver $v$ will affect the BER of the concerned MMC system with our proposed schemes in Figure 8 by setting the moving velocity as $5 \times 10^{-4} \mathrm{~m} / \mathrm{s}, 1.975 \times 10^{-4} \mathrm{~m} / \mathrm{s}, 3.95 \times 10^{-4} \mathrm{~m} / \mathrm{s}$, and $0 \mathrm{~m} / \mathrm{s}$, respectively. We can see from Figure 8 that the BER increases as the moving velocity of the receiver $v$ increases for a given drift velocity value $5 \times 10^{-4} \mathrm{~m} / \mathrm{s}$. According to the signal detection scheme in Section 3, a higher moving velocity means a more unstable position of the receiver, and the molecular concentration detected by the receiver will be more changeable. Thus, the BER increases as the moving velocity of receiver $v$ increases. We can further observe from Figure 8 that our proposed scheme can achieve a lower BER when $0 \leq v<V$ but a higher BER when $v \geq V$. This is due to the reason that when $v=0$ (i.e., the receiver is placed at a fixed position), the transmitted information molecules can always be detected in a given time period, indicating that our proposed scheme can also be applied to MC systems with a fixed receiver. When $0<v<V$ (i.e., the receiver moves at a speed lower than the drift velocity), transmitted information molecules can be detected by the receiver but within an uncertain and longer period of time due to the mobility of the receiver and the higher drift velocity. When $v=V$, the transmission of information molecules by using our proposed scheme can be reduced into the free diffusion process in [33]. As a higher ISI will be introduced during this process, a worse BER performance is achieved under this case. When $v>V$ (i.e., the receiver moves at a speed higher than the drift velocity), however, molecules can only be detected by the receiver in a very long period or in an even 
worse scenario cannot be detected by the receiver forever due to the rapid move of the receiver, the slow diffusion of the information molecules, and the lower drift velocity of the medium. Therefore, the valid range of the moving velocity $v$ with our proposed modulation and signal detection scheme is $0 \leq v<V$.

Finally, we explore how the rotational diffusion coefficient of receiver $D_{r}$ will affect the BER of the concerned MMC system with our proposed schemes in Figure 9 by setting the rotational diffusion coefficient as $1 \mathrm{rad}^{2} / \mathrm{s}, 3 \mathrm{rad}^{2} /$ $\mathrm{s}$, and $5 \mathrm{rad}^{2} / \mathrm{s}$, respectively. We can see from Figure 9 that the BER decreases as the rotational diffusion coefficient of the receiver $D_{r}$ increases. It is because $D_{r}$ represents the moving characteristic of the receiver. As $D_{r}$ increases, the receiver tends to rotate. In this case, the distance between the receiver and the transmitter tends to be constant, lower ISI will be introduced in the channel, and the concentration detected by the receiver is relatively stable. This will cause a higher successful detection of a molecule and thus a lower BER of the system. As $D_{r}$ decreases, however, the receiver tends to move to one direction. In this case, the distance between the receiver and the transmitter changes from time to time, which will introduce a higher ISI of the channel and will make it difficult for the receiver to detect the concentration peak of a molecule. This will lead to a higher BER of the system. Thus, the BER decreases as the rotational diffusion coefficient of the receiver $D_{r}$ increases.

5.3. Comparison between Our Proposed Scheme and the Available Schemes. In this subsection, we compare the BERs of our proposed schemes with the fixed threshold signal detection (FTSD) scheme [34] where the receiver uses a fixed threshold to detect signals, the low complexity signal detection (LCSD) scheme [31] where the receiver performs signal detection by judging the concavity and convexity of the concentration signals, the PAD scheme [30] where the receiver detects the signals by comparing the reconstructed peak time of current symbol with the peak time of the previous symbol, and the CATD scheme [30] where the receiver detects signals by comparing the reconstructed peak amplitude of the impulse response of current symbols with the previous reconstructed peak impulse response. Details of the CATD scheme are given by

$$
\widehat{a}_{j}= \begin{cases}1, & h_{j, \text { peak }} \geq \frac{1}{2} h_{* \text { peak }}, \\ 0, & \text { otherwise }\end{cases}
$$

where $h_{j, \text { peak }}$ is the reconstructed peak amplitude of the impulse response of the current symbol, and $h_{* \text {,peak }}$ is the peak amplitude of the impulse response of the previous symbol "1."

We plot in Figures 10-13 the BERs of the above schemes and our proposed scheme for different sequence lengths $N$, different rotational diffusion coefficients $D_{r}$, different drift velocities $V$, and different velocities of the receiver $v$, respectively. The overall experimental results show that our proposed scheme has better BER performance than these

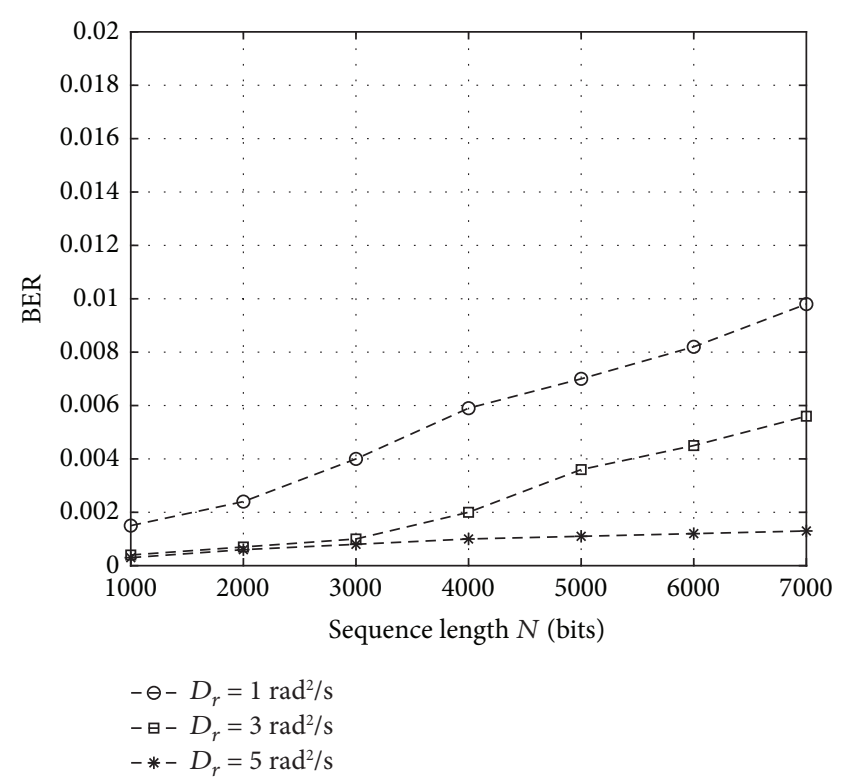

FIgURE 9: BER vs. the sequence length $N$ for different rotational diffusion coefficients $D_{r}$.

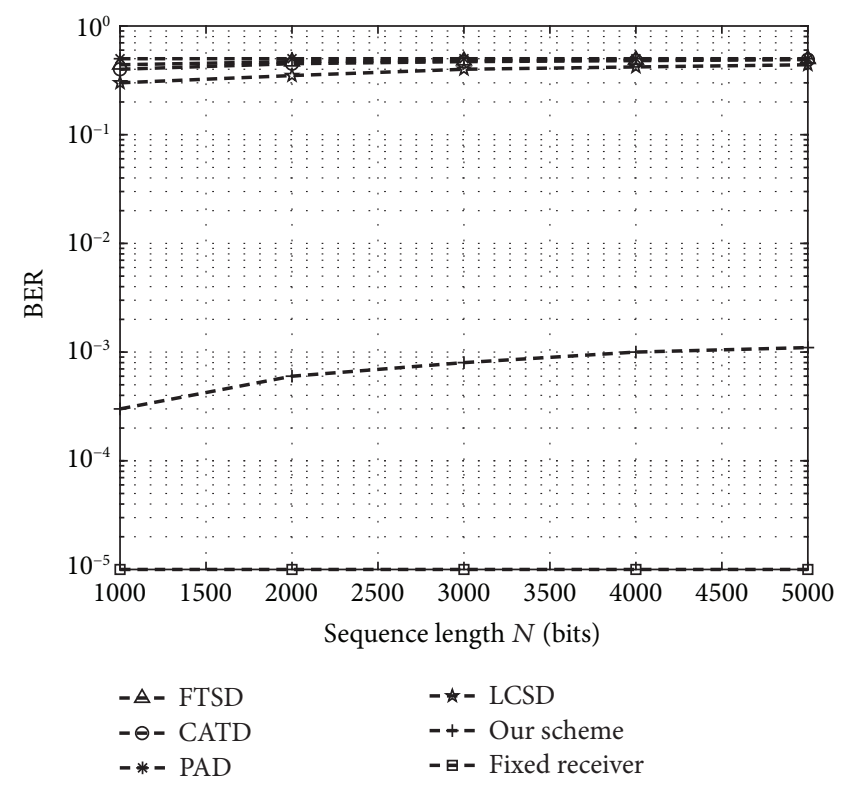

FIgURE 10: BER vs. the sequence length $N$ for different modulation and signal detection schemes.

schemes. This is because the signal detection in one block is independent of another when signals are modulated by our new modulation scheme where a third molecule $C$ is adopted to separate two different blocks. Due to this, the occurrence of a signal error in one block will affect only the signal detection in the current block and this effect will not be transferred to the signal detection in other blocks. Therefore, our signal detection scheme uses a dynamic threshold and adopts the idea of asynchronization to degrade the impact of symbol misalignment and concentration disorder caused by the mobility of the receiver on the signal detection process and thus to improve the BER 


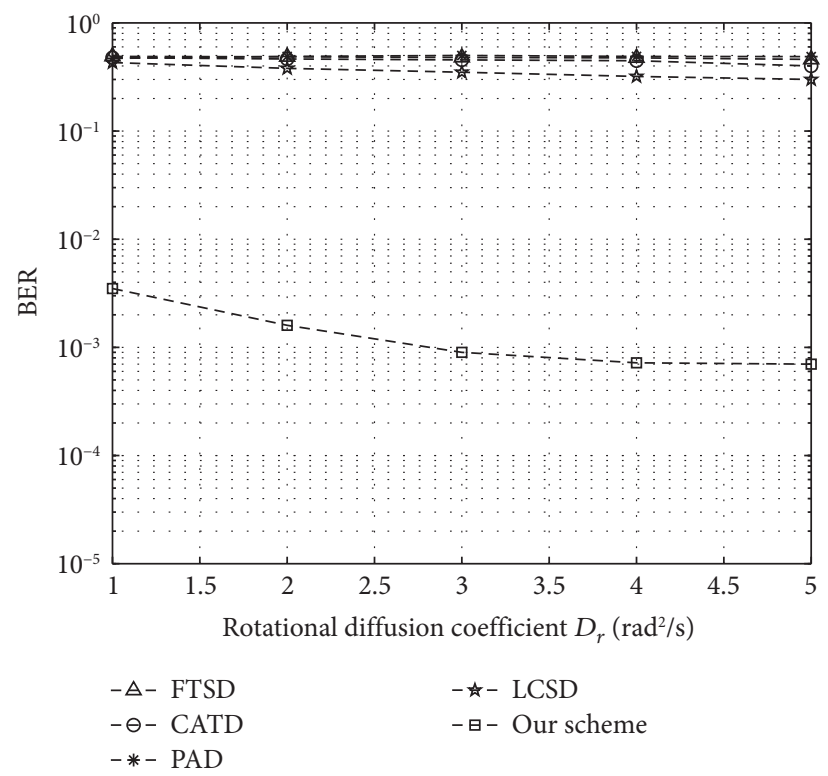

Figure 11: BER vs. the rotational diffusion coefficient $D_{r}$ for different modulation and signal detection schemes.

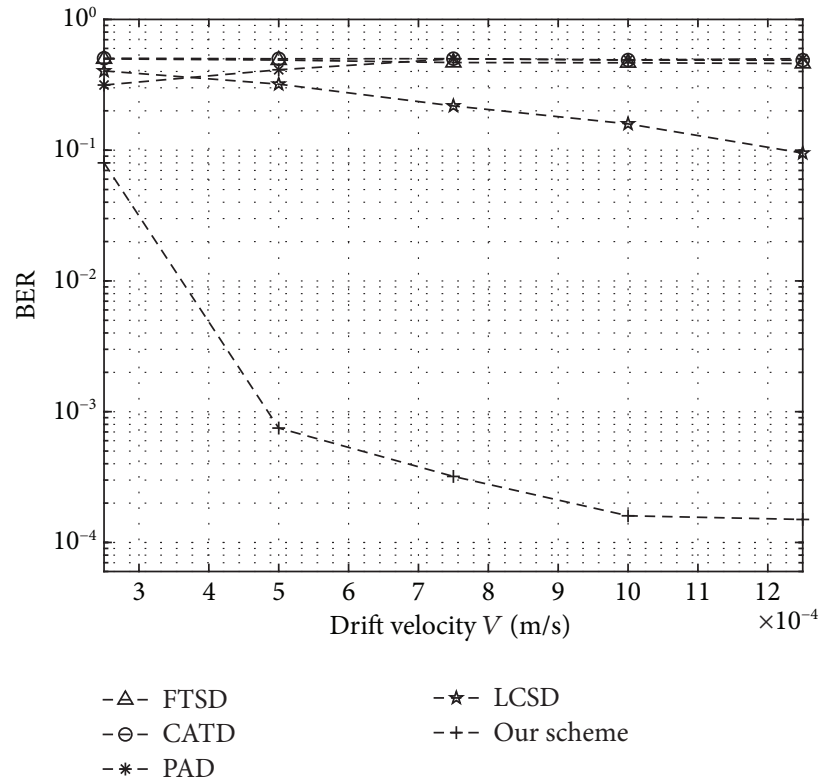

FIGURE 12: BER vs. the drift velocity of the channel media $V$ for different modulation and signal detection schemes.

performance of the concerned system. In the FTSD scheme, the CATD scheme, the PAD scheme, and the LCSD scheme, however, the symbol sequence is modulated into just one block and any signal error will greatly affect the signal detection of a whole block, which will cause a higher BER of the system. Thus, our proposed modulation and signal detection schemes can outperform these in terms of the BER. Specially, we examined in Figure 10 the BERs of our proposed scheme for MMC systems with a fixed receiver. The results showed that our scheme can achieve a lower BER performance when considering a fixed receiver. This is obvious as a lower complexity will be introduced during the signal transmission, which will result in a lower ISI of the received signals and thus a lower BER. 


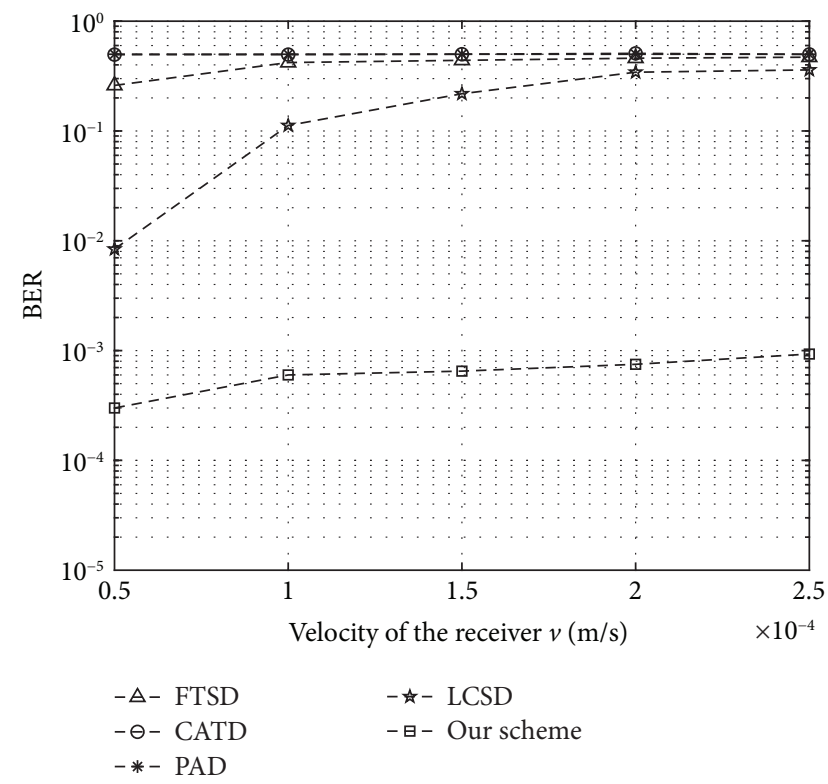

FIGURE 13: BER vs. the moving velocity of the receiver $v$ for different modulation and signal detection schemes.

\section{Conclusion}

This paper investigates the modulation and signal detection schemes for an MMC system with a fixed transmitter and a mobile receiver communicating over diffusive-drift channels. A novel modulation scheme is first proposed to address the misalignment issues in the signal detection by introducing a third molecule in the signal modulation and modulate transmitted signals into blocks of equal length. We then propose a new signal detection scheme where the distance between the transmitter and the receiver is calculated based on the idea of distance prediction and signals are detected by adopting adaptive concentration thresholds of molecules in each time interval. Finally, we conducted extensive simulations to evaluate the efficiency of our proposed schemes by the Monte Carlo simulation and compared our proposed schemes with the well-known FTSD scheme, the CATD scheme, the PAD scheme, and the LCSD scheme. The results show that our proposed schemes can achieve better
BER performance than the above schemes. Since the complexity of the diffusion process is not addressed in this paper, it serves as an interesting future research issue. Another meaningful future research issue is to study the effects of the real and estimated velocities of the receiver and the drift on the BER performance of MMC systems. As we also did not consider the channel capacity issue, studies on the channel capacity of the MMC systems and the trade-off between the channel capacity and the block length $L$ can serve as another important and interesting future research direction.

\section{Appendix}

Lemma 1. Considering the MMC system in Figure 1, the residual of molecule $C$ in the channel can be ignored as the block length $L$ is long enough.

Proof. According to the modulation rule of the proposed modulation scheme in Section 3.1, molecule $C$ is released by the transmitter at the beginning of each block to represent the start of a block transmission.

Considering the $j_{\text {th }}$ block, molecule $C$ reaches its peak concentration (i.e., the peak time) at the time [33].

$$
t^{\text {peak }}(j)=\frac{\sqrt{9 D^{2}+V^{2} d((j-1) L T)^{2}}-3 D}{V^{2}}+(j-1) L T,
$$

where $D$ is the diffusion coefficient, $V$ is the drift speed of the channel, and $d((j-1) L T)$ which is given in (6), denotes the distance between the transmitter and the receiver at the time $(j-1) L T$.

The time it takes for the molecule $\mathrm{C}$ to reach the peak concentration (i.e., the time it takes the molecule $\mathrm{C}$ to reach its concentration peak after it is released by the transmitter) is obtained as [33].

$$
Q^{\text {peak }}(j)=\frac{\sqrt{9 D^{2}+V^{2} d((j-1) L T)^{2}}-3 D}{V^{2}},
$$

and the peak concentration of molecule $\mathrm{C}$ is determined as

$$
\begin{aligned}
C\left(t^{\text {peak }}(j)-(j-1) L T\right) & =C\left(Q^{\text {peak }}(j)\right) \\
& =\frac{M}{\left(4 \pi D Q^{\text {peak }}(j)\right)^{3 / 2}} \exp \left[\frac{-\left(d((j-1) L T)-V Q^{\text {peak }}(j)\right)^{2}}{4 D Q^{\text {peak }}(j)}\right] .
\end{aligned}
$$


From the releasing of the $(j-1)_{\text {th }}$ molecule $C$ to its $j_{\text {th }}$ peak time $t^{\text {peak }}(j)$, the time it totally costs is $Q^{\text {peak }}(j)+L T$. Then, the residual concentration of the $(j-1)_{\text {th }}$ released molecule $C$ when the $j_{\text {th }}$ molecule $C$ reaches its peak concentration can be obtained as

$$
C\left(Q^{\text {peak }}(j)+L T\right)=\frac{M}{(4 \pi D(n+1) T)^{3 / 2}} \operatorname{ex}\left[\frac{-\left(d_{j(n+1)}-V(n+1) T\right)^{2}}{4 D(n+1) T}\right]
$$

Based on the parameter information and related approximation methods used in [33], we have

$$
\begin{aligned}
& \frac{C\left(Q^{\text {peak }}(j)\right)}{C\left(Q^{\text {peak }}(j)+L T\right)} \\
= & \left(\frac{Q^{\text {peak }}(j)+L T}{Q^{\text {peak }}(j)}\right)^{3 / 2} \exp \left\{\frac{(d((j-1) L T)-V P)^{2} Q^{\text {peak }}(j)}{4 D P Q^{\text {peak }}(j)}-\frac{\left((d(j-1) L T)-V Q^{\text {peak }}(j)\right)^{2} P}{4 D P Q^{\text {peak }}(j)}\right\} \\
\approx & \left(\frac{Q^{\text {peak }}(j)+L T}{Q^{\text {peak }}(j)}\right)^{3 / 2} \exp \left[L^{2} T^{2} V d(j-1) L T\right] .
\end{aligned}
$$

Since $Q^{\text {peak }}(j)+L T / Q^{\text {peak }}(j)>0$ and $L^{2} T^{2} V(d \quad(j$ $-1) L T)>0$, the residual $C$ molecules in the channel will be ignored as the block length $L$ is large enough.Here, we complete the proof.

\section{Data Availability}

The data that support the findings of this study are available from the corresponding author upon reasonable request.

\section{Conflicts of Interest}

The authors declare that they have no conflicts of interest.

\section{Acknowledgments}

This work was supported in part by the Fundamental Research Funds for the Central Universities (GK202103087), the China Postdoctoral Science Foundation (2019M663618), the Natural Science Basic Research Plan in Shaanxi Province (2020JQ-424, 2020JM-288), the National Natural Science Foundation of China (62001273, 62002210), the Xi'an Key Laboratory of Mobile Edge Computing and Security (201805052-ZD3CG36), the Major Achievements Cultivation Project of Collaborative Innovation Center of Assessment toward Basic Education Quality at Beijing Normal University (2020-05-0034-BZPK01), and the Open Project Program of the Shaanxi Key Laboratory for Network Computing and Security Technology (NCST2021YB-02).

\section{References}

[1] O. D. Kose, M. C. Gursoy, M. Saraclar, A. E. Pusane, and T. Tugcu, "Machine learning-based silent entity localization using molecular diffusion," IEEE Communications Letters, vol. 24, no. 4, pp. 807-810, 2020.

[2] C. Lee, B. Koo, and C. Chae, "Demo: in-vessel molecular MIMO communications," in Proceedings of the IEEE Wireless Communication Network Conference Workshops, pp. 1-2, Seoul, South Korea, May 2020.

[3] W. Guo, T. Asyhari, N. Farsad et al., "Molecular communications: channel Model and physical layer techniques," IEEE Wireless Communications, vol. 23, no. 4, pp. 120-127, 2016.

[4] P. Kulakowski, K. Turbic, and L. M. Correia, "From nanocommunications to body area networks: a perspective on truly personal communications," IEEE Access, vol. 8, pp. 159839159853, 2020.

[5] R. Zheng, L. Lin, and H. Yan, "A noise suppression filter for molecular communication via diffusion," IEEE Wireless Communications Letters, vol. 10, no. 3, pp. 589-593, 2021.

[6] K. Yang, D. Bi, Y. Deng et al., "A comprehensive survey on hybrid communication in context of molecular communication and terahertz communication for body-centric nanonetworks," IEEE Transactions on Molecular, Biological and Multi-Scale Communications, vol. 6, no. 2, pp. 107-133, 2020.

[7] A. Al-Helali, B. Liang, and N. Nasser, "Novel molecular signaling method and system for molecular communication in human body," IEEE Access, vol. 8, pp. 119361-119375, 2020.

[8] N. Pandey, S. Joshi, R. K. Mallik, and B. Lall, "Channel characterization for devices in a turbulent diffusive environment: a mobile molecular communication approach," 
IEEE Transactions on Molecular, Biological and Multi-Scale Communications, vol. 5, no. 3, pp. 222-232, 2019.

[9] T. Suda and T. Nakano, "Molecular communication as a biological system," in Proceedings of the IEEE International Conference on Sensing, Communication and Networking (SECON Workshops), pp. 1-4, Hong Kong, China, June 2018.

[10] A. Guney, B. Atakan, and O. B. Akan, "Mobile ad hoc nanonetworks with collision-based molecular communication," IEEE Transactions on Mobile Computing, vol. 11, no. 3, pp. 353-366, 2012.

[11] Y. Lu, R. Ni, and Q. Zhu, "Wireless communication in nanonetworks: current status, prospect and challenges," IEEE Transactions on Molecular, Biological and Multi-Scale Communications, vol. 6, no. 2, pp. 71-80, 2020.

[12] A. Ahmadzadeh, V. Jamali, and R. Schober, "Stochastic channel modeling for diffusive mobile molecular communication systems," IEEE Transactions on Communications, vol. 66, no. 12, pp. 6205-6220, 2018.

[13] I. F. Akyildiz, A. Kak, and S. Nie, " $6 \mathrm{~g}$ and beyond: the future of wireless communications systems," IEEE Access, vol. 8, pp. 133995-134030, 2020.

[14] T. N. Cao, A. Ahmadzadeh, V. Jamali et al., "Diffusive mobile MC with absorbing receivers: stochastic analysis and applications," IEEE Transactions on Molecular, Biological and Multi-Scale Communications, vol. 5, no. 2, pp. 84-99, 2019.

[15] S. Solak and M. Oner, "Sequential decision fusion for abnormality detection via diffusive molecular communications," IEEE Communications Letters, vol. 25, no. 3, pp. 825-829, 2021.

[16] N. Sharifi, Y. Zhou, G. Holmes, and Y. Chen, "Overcoming channel uncertainties in touchable molecular communication for direct-drug-targeting-assisted immuno-chemotherapy," IEEE Transactions on NanoBioscience, vol. 19, no. 2, pp. 249-258, 2020.

[17] A.-A. A. Boulogeorgos, S. E. Trevlakis, S. A. Tegos, V. K. Papanikolaou, and G. K. Karagiannidis, "Machine learning in nano-scale biomedical engineering," IEEE Transactions on Molecular, Biological and Multi-Scale Communications, vol. 7, no. 1, pp. 10-39, 2021.

[18] N. R. Kim and C. B. Chae, "Novel modulation techniques using isomers as messenger molecules for nano communication networks via diffusion," IEEE Journal on Selected Areas in Communications, vol. 31, no. 12, pp. 847-856, 2014.

[19] B. Krishnaswamy, C. M. Austin, J. P. Bardill et al., "Timeelapse communication: bacterial communication on a microfluidic chip," IEEE Transactions on Communications, vol. 61, no. 12, pp. 5139-5151, 2013.

[20] M. S. Kuran, H. B. Yilmaz, T. Tugcu, and I. F. Akyildiz, "Modulation techniques for communication via diffusion in nanonetworks," in Proceedings of the IEEE International Conference on Communications (ICC), pp. 1-5, Kyoto, Japan, June 2011.

[21] M. H. Kabir, S. M. Riazul Islam, and K. S. Kwak, "D-MoSK modulation in molecular communications," IEEE Transactions on NanoBioscience, vol. 14, no. 6, pp. 680-683, 2015.

[22] J. Wang, X. Liu, M. Peng, and M. Daneshmand, "Performance analysis of D-MoSK modulation in mobile diffusive-drift molecular communications," IEEE Internet of Things Journal, vol. 7, no. 11, pp. 11318-11326, 2020.

[23] S. Huang, L. Lin, W. Guo, H. Yan, J. Xu, and F. Liu, "Initial distance estimation and signal detection for diffusive mobile molecular communication," IEEE Transactions on NanoBioscience, vol. 19, no. 3, pp. 422-433, 2020.
[24] N. Varshney, A. Patel, W. Haselmayr, A. K. Jagannatham, P. K. Varshney, and A. Nallanathan, "Impact of intermediate nanomachines in multiple cooperative nanomachine-assisted diffusion advection mobile molecular communication," IEEE Transactions on Communications, vol. 67, no. 7, pp. 48564871, 2019.

[25] L. Chouhan, P. K. Sharma, and N. Varshney, "Optimal transmitted molecules and decision threshold for drift-induced diffusive molecular channel with mobile nanomachines," IEEE Transactions on NanoBioscience, vol. 18, no. 4, pp. 651-660, 2019.

[26] Y. Okaie, T. Nakano, T. Hara et al., "Cooperative target tracking by a mobile bionanosensor network," IEEE Transactions on NanoBioscience, vol. 13, no. 3, pp. 267-277, 2014.

[27] P. He, B. Pi, and Q. Liu, "Calcium signaling in mobile molecular communication networks: from a multimedia view," IEEE Access, vol. 7, no. 99, pp. 164825-164834, 2019.

[28] S. Qiu, T. Asyhari, and W. Guo, "“Mobile molecular communications: positional-distance codes," in Proceedings of the IEEE 17th International Workshop on Signal Processing Advances in Wireless Communications (SPAWC), pp. 1-5, Edinburgh, UK, July 2016.

[29] Z. Luo, L. Lin, and M. Ma, "Offset estimation for clock synchronization in mobile molecular communication system," in Proceedings of the IEEE Wireless Communications and Networking Conference, pp. 1-6, Doha, Qatar, April 2016.

[30] G. Chang, L. Lin, and H. Yan, "Adaptive detection and ISI mitigation for mobile molecular communication," IEEE Transactions on NanoBioscience, vol. 17, no. 99, pp. 21-35, 2018.

[31] X. Mu, H. Yan, B. Li et al., "Low-complexity adaptive signal detection for mobile molecular communication," IEEE Transactions on NanoBioscience, vol. 19, no. 2, pp. 237-248, 2020.

[32] L. Felicetti, M. Femminella, and G. Reali, "Establishing digital molecular communications in blood vessels," in Proceedings of the First International Black Sea Conference on Communications and Networking (BlackSeaCom), pp. 54-58, Batumi, Georgia, July 2013.

[33] L. Lin, Q. Wu, M. Ma, and H. Yan, "Concentration-based demodulation scheme for mobile receiver in molecular communication," Nano Communication Networks, vol. 20, pp. 11-19, 2019.

[34] M. U. Mahfuz, D. Makrakis, and H. T. Mouftah, "On the characterization of binary concentration-encoded molecular communication in nanonetworks," Nano Communication Networks, vol. 1, no. 4, pp. 289-300, 2010.

[35] H. C. Berg, Random Walks in Biology, Princeton University Press, Princeton, NJ, USA, 1993.

[36] N. Bouzakaria, C. Concolato, and J. L. Feuvre, "Overhead and performance of low latency live streaming using MPEGDASH," in Proceedings of the 5th International Conference on Information, Intelligence, Systems and Applications, pp. 1-6, Chania, Greece, July 2014.

[37] M. Moore and T. Nakano, "Comparing transmission, propagation, and receiving options for nanomachines to measure distance by molecular communication," in Proceedings of the 2012 IEEE International Conference on Communications (ICC), pp. 6132-6136, Ottawa, Canada, June 2012.

[38] M. Moore, T. Nakano, A. Enomoto, and T. Suda, "Measuring distance from single spike feedback signals in molecular communication," IEEE Transactions on Signal Processing, vol. 60, no. 7, Article ID 35763587, 2012. 
[39] A. Noel, K. C. Cheung, and R. Schober, "Bounds on distance estimation via diffusive molecular communication," in Proceedings of the IEEE GLOBECOM, Austin, TX, USA, December 2014.

[40] Y. Chen, Y. Li, L. Lin, and H. Yan, "Parameter estimation of diffusive molecular communication with drift," IEEE Access, vol. 8, pp. 142704-142713, 2020.

[41] J. Huang, H. Lai, Y. Lee, C. Lee, and P. Yeh, "Distance estimation in concentration-based molecular communications," in Proceedings of the IEEE GLOBECOM, Atlanta, USA, December 2013.

[42] X. Wang, M. Higgins, and M. Leeson, "Distance estimation schemes for diffusion based molecular communication systems," IEEE Communication Letter, vol. 19, no. 3, Article ID 399402, 2015.

[43] D. Kilinc and O. B. Akan, "Receiver design for molecular communication," IEEE Journal on Selected Areas in Communications, vol. 31, no. 12, pp. 705-714, 2013.

[44] A Simulator of Modulation and Signal Detection for Molecular Communication with a Mobile Receiver, https://www. researchgate.net/publication/35666256.

[45] M. Gregori and I. Akyildiz, "A new nanonetwork architecture using flagellated bacteria and catalytic nanomotors," IEEE Journal on Selected Areas in Communications, vol. 28, no. 4, pp. 612-619, 2010.

[46] Y. Okaie, T. Nakano, T. Obuchi, T. Hara, and S. Nishio, "Bacterium-based mobile mionanosensor networks for target tracking: a biologically realistic model," in Proceedings of the ACM the 8th International Conference on Bioinspired Information and Communications Technologies, pp. 188-190, Boston, MA, USA, December 2014.

[47] L. Felicetti, M. Femminella, and G. Reali, "A molecular communications system for the detection of inflammatory levels related to COVID-19 disease," IEEE Transactions on Molecular, Biological and Multi-Scale Communications, vol. 7, no. 3, pp. 165-174, 2021.

[48] M. Pierobon and I. F. Akyildiz, "Capacity of a diffusion-based molecular communication system with channel memory and molecular noise," IEEE Transactions on Information Theory, vol. 59, no. 2, pp. 942-954, 2013.

[49] C. Rose, I. S. Mian, and M. Ozmen, "Capacity bounds on point-to-point communication using molecules," Proceedings of the IEEE, vol. 107, no. 7, pp. 1342-1355, 2019. 